\title{
Modelo para estimativa de força e torque dos músculos rotadores externos do ombro no plano transverso
}

\author{
Marcelo P. Castro \\ Felipe O. Marques \\ Juliana M. Costa \\ Joelly M. Toledo \\ Roberto C. Krug \\ Daniel C. Ribeiro \\ Jefferson F. Loss
}

\author{
Universidade Federal do Rio Grande do Sul \\ Escola Superior de Educação Física \\ Porto Alegre \\ Brasil
}

\section{RESUMO}

Estimar a força dos músculos do ombro e compreender o quanto cada músculo contribui para produção do torque em determinado movimento articular torna-se indispensável para a compreensão do movimento humano, bem como para o planejamento da reabilitação de pacientes com disfunções no ombro. Neste sentido, o presente estudo tem como principal objetivo estimar a força muscular e analisar a contribuição dos músculos Infra-espinhoso, Redondo Menor, Supra-espinhoso,

Deltóide Posterior e Deltóide Médio no torque de rotação externa dinâmico do ombro no plano transverso. Para isso, um modelo de otimização gerado no software MATLAB, 7.0 (MathWork Inc, Massachusetts - USA), capaz de estimar a produção de força e torque de cada um dos músculos rotadores externos do ombro foi utilizado.Os músculos Infra-espinhoso e Redondo Menor apresentaram um pico de torque de $22 \mathrm{Nm}$ em $20^{\circ}$ e $6 \mathrm{Nm}$ em $28^{\circ}$, respectivamente, quando o ombro encontrava-se em rotação interna. A magnitude máxima de força alcançada por estes músculos foi de $996 \mathrm{~N}$ para o

Infraespinhoso e 306N para o Redondo Menor. Os músculos Supra-espinhoso, Deltóide Posterior e Deltóide Médio praticamente não produziram torque e obtiveram magnitudes pequenas de força. Estes resultados apontam que os músculos Infraespinhoso e Redondo Menor são preferencialmente recrutados para o gesto de rotação externa do ombro.

Palavras-chave: ombro, torque muscular, força muscular e modelo de otimização.

\section{ABSTRACT \\ Model for force and moment prediction of shoulder external rotation muscles in the transverse plane}

Estimating shoulder muscle forces and understanding the contribution of each muscle to the torque generated during joint movement is indispensable for the knowledge of the human movement, as well as, for the rehabilitation planning of patients with shoulder dysfunction. For this reason, the goal of the present study is to estimate the muscle force and to analyze the contribution of the following muscles: Infraspinatus, Teres Minor, Supraspinatus, Deltoideus Posterior and Deltoideus Medialis during dynamic external rotation of the shoulder in the transverse plane. So, an optimization model structured in the software MATLAB 7.0 (MathWork Inc., Massachusetts - the USA) was used to estimate the force and moment production of each the shoulder external rotators muscles. The muscles Infraspinatus and Teres Minor reached the following peak moment, respectively: $22 \mathrm{Nm}\left(20^{\circ}\right.$ of internal rotation) and $6 \mathrm{Nm}$ (28 of internal rotation). The force magnitudes reached by these muscles were $996 \mathrm{~N}$ for the Infraspinatus and $306 \mathrm{~N}$ for the Teres Minor, both at $31^{\circ}$ of internal rotation. The muscles Supraspinatus, Deltoideus Posterior and Deltoideus Medialis presented low magnitudes for external rotation moment and force. These results suggested Infraspitatus and Teres Minor muscles are preferentially recruited during external rotation of the shoulder.

Key-words: shoulder, moment, muscle force and optimization model 


\section{INTRODUÇÃ̃o}

O ombro é a mais móvel articulação do corpo humano, possui três graus de liberdade, o que permite orientar o membro superior em relação aos três planos no espaço(27). Observa-se somente um pequeno grau de estabilidade oferecido pelas estruturas ósseas e, por conseqüência disto, a estabilidade do ombro é consideravelmente dependente dos tecidos moles ${ }^{(32,50)}$. Dentre estes tecidos, os músculo são considerados os principais estabilizadores $(24,44)$. O torque do músculo determina sua capacidade de mover uma articulação, é definido como o produto entre força muscular e distância perpendicular $\left(\mathrm{d}^{\perp}\right)$. Músculos com maiores $\mathrm{d}^{\perp}$ geram mais torque quando é produzida uma mesma força. Assim, a $\mathrm{d}^{\perp}$ representa a vantagem mecânica do músculo sobre a articulação(14, 24, 37, 45, 48).

As forças internas que agem sobre o corpo humano são: forças musculares, articulares e ligamentares(2). As forças internas podem ser determinadas diretamente por meio de implantação de transdutores de forças ou por meio de modelos biomecânicos. Estes modelos consistem de algoritmos que processam informações iniciais (dados de entrada) utilizando critérios mecânicos e/ou fisiológicos para obtenção de forças (dados de saída). Medidas de antropometria, cinemetria e dinamometria são usualmente utilizadas como dados de entrada(51).

Outros estudos já foram realizados visando mensurar a produção de força e torque dos músculos do

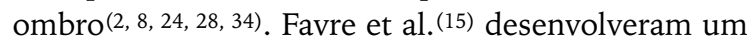
algoritmo para estimar a força muscular a partir de um modelo biomecânico tridimensional . Da mesma forma, Hughes e An (24) e Karlsson e Peterson(28) também investigaram a força muscular utilizando um modelo biomecânico tridimensional. Uma outra forma de operar com modelos biomecânicos, é através do uso do sinal eletromiográfico em associação com dados de torque isocinético para identificar padrões de ativação de força muscular durante a rotação interna (RI) e rotação externa (RE) do ombro(8). Modelos probabilísticos, os quais calculam a distribuição das forças entre os músculos a partir de dados de entrada, também são utilizados para investigar a produção de força máxima dos músculos rotadores externos do ombro( ${ }^{(34)}$. Entretanto, não foram localizados na literatura trabalhos que apre- sentem a força e o torque muscular de cada rotador externo ao longo da amplitude de movimento (ADM) durante o movimento dinâmico.

Assim, por existir uma carência de estudos investigando força e torque muscular durante atividade dinâmica, o presente estudo tem como principal objetivo estimar a força e o torque muscular e analisar a contribuição dos músculos Infra-espinhoso (IE), Redondo Menor (Rm), Supra-espinhoso (SE), Deltóide Posterior (DP) e Deltóide Médio (DM) para o torque de RE dinâmico do ombro no plano transverso.

\section{MATERIAIS E MÉTODOS}

Para a realização deste trabalho, foram utilizados dados referentes ao torque de RE do ombro no plano transverso, coletados em um dinamômetro isocinético da marca Cybex, modelo Norm (Dataq Instruments, Inc. Ohio - EUA). Foi utilizado junto ao dinamômetro um eletrogoniômetro da marca Biomectrics Ltd (Cwmfelinfach, Reino Unido, modelo XM 180) com o objetivo de registrar a posição articular em função do tempo, com uma taxa de amostragem que permite melhor definição espacial do que a fornecida pelo dinamômetro. Ambos os aparelhos, estavam conectados a um microcomputador Pentium III $650 \mathrm{MHz}$ através de um conversor analógico-digital da marca Computer Boards (modelo CIODAS 16, 16 bits) a uma taxa de aquisição de 2000 $\mathrm{Hz}$, sendo assim, a aquisição dos dados foi realizada de maneira simultânea, dentro de uma mesma base temporal, não necessitando de sincronismo. Foram utilizados para tratamento dos dados o software SAD32 (Sistema de Aquisição de Dados, desenvolvido pelo Laboratório de Medições Mecânicas da Universidade Federal do Rio Grande do Sul) e o software MATLAB 7.0 ( (MathWork Inc, Massachusetts - USA). Os sinais de torque e ângulo foram tratados com um filtro digital, Butterworth, passa baixa, ordem 3 com freqüência de corte de $3 \mathrm{~Hz}$, para os dados de ângulo, e $10 \mathrm{~Hz}$, para dados de torque. A população do estudo foi jovens universitários de Porto Alegre, os quais foram convidados diretamente pelos pesquisadores, ao acaso, para participarem do estudo. A amostra foi constituída de 8 indivíduos voluntários do sexo masculino, todos não eram atletas mas praticantes de atividade física regular ( 2 ou 3 vezes por semana), apresentavam idade entre 19 e 25 
anos, massa corporal média de $70 \mathrm{~kg}( \pm 6 \mathrm{~kg})$ e altura média de $170 \mathrm{~cm}( \pm 10 \mathrm{~cm})$. O ombro avaliado foi o direito (dominante), sendo que os indivíduos seriam excluídos do estudo se fossem sedentários ou atletas ou se apresentassem qualquer anormalidade ou histórico de lesão no ombro avaliado. Todos os indivíduos que participaram dessa pesquisa concordaram por livre arbítrio e assinaram o Termo de Consentimento Livre e Esclarecido, aprovado pelo comitê de Ética da Universidade onde foi realizado o estudo.

Os procedimentos de coleta constituíram-se de cinco fases: preparação, posicionamento, calibração, familiarização com o teste e teste. Durante a fase de preparação, os indivíduos realizaram aquecimentos e alongamentos dos membros superiores. Após, os indivíduos foram posicionados no dinamômetro isocinético: em ortostase, com o membro superior direito em posição neutra (junto ao tronco) e cotovelo em flexão de 90 graus. Esta posição foi escolhida por ser a mais usual para reforço dos músculos do manguito rotador na reabilitação do ombro. Durante a familiarização, os indivíduos realizaram três repetições de contracções concêntricas submáximas de RE e RI. Durante o teste cada participante realizou cinco repetições de contracções concêntricas máximas de RE e RI sem intervalo, em uma velocidade angular de $60^{\circ} / \mathrm{s}$. Após a filtragem dos sinais, foram feitas as médias das cinco repetições.

Os valores de $\mathrm{d}^{\perp} \mathrm{e}$ área de secção transversa fisiológica (ASTF) adotados no modelo foram àqueles apresentados por Kuechle et al.(33), conforme mostra a Tabela 1. A faixa etária do estudo de Kuechle et al.(33), o qual foi realizado em cadáveres, varia de 38 a 80 anos diferindo da faixa etária do presente estudo, entretanto, provavelmente devido à dificuldade de realizar estudos com medições diretas, não foi encontrado na literatura outro estudo que apresentasse tais dados com amostra mais semelhante a do presente estudo. Além disso, acredita-se que os valores de $\mathrm{d}^{\perp}$, visto que em ambas amostras os indivíduos não estavam mais em fase de crescimento, a idade não acarrete em grandes diferenças nesta medida, visto que é uma característica mecânica. Relativamente à ASTF, as alterações provenientes da idade ocorrerão em todos os músculos estudados, diminuindo assim a influência que tais fatores poderiam apresentar para o estudo.
A tensão específica ( $\sigma)$ ou tensão muscular máxima é uma constante, utilizada para estabelecer os limites de força máxima que o músculo é capaz de produ$\operatorname{zir}(5,6,28,43,53)$, expressando a capacidade de produção de força muscular por unidade de área. $\mathrm{O}$ valor de $\mathrm{s}$ adotado neste trabalho foi de $70 \mathrm{~N} / \mathrm{cm}^{2}(53)$. Baseado no algoritmo desenvolvido por Favre et al.(15), o modelo assume que o torque mensurado pelo dinamómetro isocinético é resultado do somatório dos torques produzidos pelos músculos rotadores externos (Equação 1). Em um primeiro momento, o modelo busca equilibrar esta equação com os motores primários, posteriormente, o modelo inclui os motores secundários.

Para determinação dos músculos motores primários e secundários, inicialmente o modelo calcula o torque potencial de cada músculo. Torque potencial é produto da $\mathrm{d}^{\perp}$ pela força potencial. Força potencial é produto da ASTF do músculo pela constante de ten-

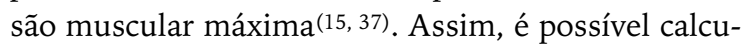
lar a força máxima que o músculo, teoricamente, pode produzir e consequentemente, o torque máximo que ele é capaz de gerar em cada ângulo da ADM. Feito isso, a média entre os picos de torques potenciais de cada um dos músculos é calculada e multiplicada por um fator limiar de $0,7(15)$. Este valor calculado servirá como factor limiar para determinação dos motores primários e secundários. Desta forma, os músculos que apresentarem um valor de torque potencial médio acima do valor calculado serão considerados motores primários, caso contrário, serão motores secundários.

O torque de RE mensurado pelo dinamômetro isocinético é resultado do somatório dos torques produzidos pelos músculos rotadores externos, conforme Equação (1):

$$
T M=\sum_{i=1}^{n} T m u s c_{i}
$$

$i=1,2,3, \ldots$ nésimo músculo rotador externo;

$T M=$ torque mensurado no dinamómetro;

Tmusc $=$ torque do músculo rotador externo;

Assumindo que a força muscular máxima é o produto entre ASTF e o ${ }^{(37)}$, um músculo (u) de maior ASTF foi escolhido e a força muscular deste músculo foi calculada, através da Equação (2): 


$$
F u=\sigma \cdot A S T F u
$$

$F u=$ força do músculo $(u)$;

$\sigma=$ tensão específica;

$A S T F u$ = área de secção transversa fisiológica do músculo (u);

O músculo (u) também foi considerado como referência para normalização das ASTF através da determinação de um quociente $\left(Q_{i}\right)$, a exemplo do algoritmo proposto por Favre et al. (15), tal procedimento foi adotado somente com o intuito de deixar o processamento dos dados mais rápido. Tal cálculo é expresso pela Equação (3):

$$
Q_{i}=A S T F_{i} / A S T F u
$$

$i=1,2, \ldots$ nésimo músculo rotador externo;

$Q_{i}=$ quociente do músculo $\mathrm{i}$;

$A S T F_{i}=$ área de secção transversa fisiológica do músculo i;

Visto que o torque muscular é o produto da força muscular pela $\mathrm{d}^{\perp}(12,24,37,45)$, após a determinação de $Q_{i}$ e $F u$ a soma dos torques musculares produzidos pelos motores primários pode ser estimada por meio da Equação (4). Assim, as variações da magnitude dos torques musculares (Tmusc) são obtidas pelas variações de $F u$. A variação de $F u$ pode ser feita com diferentes magnitudes de intervalo, sendo esta magnitude denominada de incremento $(\ddot{A})$. O modelo realiza variações do $\ddot{A}$ de 0 a $85 \%$ visto que, os músculos não são capazes de produzir uma tensão máxima por unidade de área(52). Segundo Edman et al.(13), durante a contração voluntária máxima, a ativação muscular quase nunca é verdadeiramente máxima. Neste modelo, o $\ddot{A}$ adotado foi de $1 \%$. Portanto, o modelo permite 86 possibilidades de Tmusc, com a aplicação da Equação (4):

$$
T M=\ddot{A} \cdot F u^{M P} \cdot\left(1 \cdot d u^{\perp}+Q_{i} \cdot d_{i}{ }^{\perp}+\ldots Q_{n} \cdot d_{n}{ }^{\perp}\right)
$$

$T M=$ torque mensurado no dinamômetro;

$\Delta \Delta=0 ; 0,01 ; 0,02 \ldots .0,85$;

$F u^{M P}=$ força do músculo $(\mathrm{u})$ para os motores primários;

$d^{\perp} \mathrm{u}=$ distância perpendicular do músculo (u); $i=1,2, \ldots$ nésimo músculo motor primário;

$Q_{, i}=$ quociente dos músculos motores primários;

$d^{\perp}{ }_{i}=$ distância perpendicular dos músculos motores primários;

O modelo busca encontrar qual combinação de torques musculares, somando inicialmente os músculos considerados motores primários, produz um valor de Tmusc mais próximo do valor de TM. A diferença entre o TM e o Tmusc dos motores primários denomina-se Erro Mínimo $1\left(\mathrm{EM}_{1}\right)$. Então, o valor de $F u$ responsável pelo Tmusc dos motores primários com menor $\mathrm{EM}_{1}$ é armazenado, esse processo é repetido para cada ângulo da ADM. Posteriormente, o $E_{1}$ é utilizado conforme demonstra a Equação (5), para distribuir o torque restante entre os músculos motores secundários do movimento.

$$
E M_{1}=\ddot{A} \cdot F u^{M S} \cdot\left(Q_{i} \cdot d_{i}{ }^{\perp} Q_{i} \cdot d_{i}{ }^{\perp}+\ldots Q_{n} \cdot d_{n}{ }^{\perp}\right)
$$

$E M_{1}=$ erro mínimo 1 ;

$F u^{M S}=$ força do músculo $(u)$ para os motores secundários;

$i=1,2, \ldots$ nésimo músculo motor secundário;

$Q_{i}=$ quociente dos músculos motores secundários;

$\mathrm{d}^{\perp} i=$ distância perpendicular dos músculos motores secundários;

A Equação (5) busca encontrar qual combinação de torque, somada entre os músculos considerados motores secundários, produz um valor de Tmusc mais próximo ao $\mathrm{EM}_{1}$. A diferença entre o Tmusc dos motores secundários e o $\mathrm{EM}_{1}$ denomina-se Erro Mínimo $2\left(\mathrm{EM}_{2}\right)$. O valor de $F u$ responsável pelo Tmusc dos motores secundários com menor $\mathrm{EM}_{2}$ é armazenado, esse processo é repetido para cada ângulo da ADM. Desta forma, o $\mathrm{EM}_{2}$ é o torque que o modelo não foi capaz de equilibrar.

Assim, com os valores de $F u^{M P}$ armazenados é possível estimar, através da Equação (6), qual a força que cada músculo motor primário produziu em cada ângulo.

$$
F_{i}=Q_{i} \cdot F u^{M P}
$$

$i=1,2, \ldots$ nésimo músculo motor primário;

$F_{i}=$ força do músculo i; 
$Q_{i}=$ quociente do músculo i;

$F u^{M P}=$ força do músculo (u) armazenada para cada ângulo correspondente aos motores primários;

E a partir dos valores de $F u^{\text {MS }}$ armazenados é possível estimar, através da Equação (7), qual a força que cada músculo motor secundário produziu em cada ângulo.

$$
F_{i}=Q_{i} \cdot F u^{M S}
$$

$i=1,2, \ldots$ nésimo músculo motor secundário;

$F_{i}=$ força do músculo i;

$Q_{i}=$ quociente do músculo i;

$F u^{\mathrm{MS}}=$ força do músculo (u) armazenada para cada ângulo correspondente aos motores secundários;

Estimando a força produzida por cada músculo, é possível estimar o torque que cada músculo produziu, utilizando a Equação (8):

$$
T_{i}=F_{i} \cdot d^{\perp}
$$

$i=1,2, \ldots$ nésimo músculo rotador externo;

$T_{i}=$ torque do músculo i;

$d^{\perp}{ }_{i}=$ distância perpendicular do músculo i;

\section{Análise Estatística}

Para avaliar estatisticamente eventuais diferenças entre as forças obtidas para os vários músculos, foi calculada a diferença dos valores de força entre cada dois músculos em cada grau da amplitude de movimento, resultando em um novo conjunto de valores. Valores próximos a zero representam desta forma, similaridade entre dois músculos, ao passo que quanto maior for a diferença entre os músculos, maiores serão os valores. A normalidade deste novo conjunto de valores foi verificada através do teste de Kolmogorov-Smirnov com a correcção de Lilliefors. As diferenças foram calculadas entre os músculos IE e $\mathrm{Rm}$, IE e SE e Rm e SE. Estes músculos foram escolhidos porque o IE e o Rm são os músculos que apresentaram maiores magnitudes de força e torque. Visto que o SE, o DM e o DP apresentaram magnitudes muito similares de força e torque, o SE foi escolhido de maneira aleatória, para representar esse conjunto de músculos, isto porque possivelmente diferenças encontradas entre o SE e os outros mús- culos também seriam encontradas entre o DM e o DP se comparados a estes mesmos músculos. Tal procedimento também foi realizado com os dados de torque. Assim, 6 novos conjuntos de valores foram obtidos (3 referentes às diferenças dos valores de força entre os 3 pares de músculos escolhidos e 3 referentes às diferenças entre estes mesmos 3 pares de músculos para os dados de torque). Em um segundo momento, foi realizada uma análise descritiva destes 6 conjuntos de valores. A partir desta análise foi estabelecido um valor que expressasse a diferença entre cada par de músculos escolhido (cada novo conjunto de valores) ao longo de toda amplitude de movimento. Este valor foi denominado valor estabelecido (VE), sendo assim, 6 VEs foram encontrados. Foi utilizado o teste $t$-student para comparar o conjunto de valores que representa a diferença entre cada par de músculos com o seu respectivo VE. Possibilitando assim, conhecer o quanto, no mínimo, um músculo contribui a mais que o outro ao longo de toda amplitude de movimento. O nível de significância utilizado em todos os testes foi $\mathrm{p}<0,05$. Os procedimentos estatísticos foram realizados no programas SPSS, versão 13.0.

\section{RESULTADOS}

A média do torque mensurado no dinamômetro foi de $24 \mathrm{Nm}( \pm 7,7)$, alcançando um valor de pico de $28 \mathrm{Nm}$ em $30^{\circ}$ de RI.

Tabela 1. Valores de dffi, ASTF, Torque (Potencial Médio, Média, Desvio Padrão e Pico/Ângulo] e Força (Média, Desvio Padrão e Pico/Ângulo)-

\begin{tabular}{lccccc}
\hline & IE & Rm & DM & SE & DP \\
\hline $\mathrm{d}^{\perp}$ pico $(\mathrm{cm})$ & 2,55 & 2,17 & 0,09 & 0,57 & 0,46 \\
\hline ASTF $\left(\mathrm{cm}^{2}\right)$ & 9,51 & 2,92 & 9,08 & 5,21 & 9,45 \\
\hline Torque $(\mathrm{Nm})$ & & & & & \\
\hline Potencial Médio & 26.9 & 7 & 0.6 & 2.6 & 5.5 \\
\hline Média & 18,54 & 4,94 & 0,00 & 0,02 & 0,05 \\
\hline Desvio Padrão & 2,44 & 0,98 & 0,01 & 0,01 & 0,02 \\
\hline Pico (Ângulo) & $22\left(32^{\circ}\right)$ & $6\left(44^{\circ}\right)$ & $0,01\left(18^{\circ}\right)$ & $0,04\left(50^{\circ}\right)$ & $0,08\left(2^{\circ}\right)$ \\
\hline Força (N) & & & & & \\
\hline Média & 802,64 & 246,5 & 12,12 & 6,95 & 12,64 \\
\hline Desvio Padrão & 136,33 & 41,84 & 4,40 & 2,59 & 4,54 \\
\hline Pico (Ângulo) & $997\left(31^{\circ}\right)$ & $306\left(33^{\circ}\right)$ & $19\left(37^{\circ}\right)$ & $11\left(41^{\circ}\right)$ & $20\left(37^{\circ}\right)$ \\
\hline
\end{tabular}

$\left(d^{\perp}=\right.$ distância perpendicular; ASTF=área de secção transversa fisiológica; $I \mathcal{E}=$ infra-espinhoso; $R m$ = redondo menor; $S \mathcal{E}=$ supra-espinhoso; $D P=$ deltóide posterior; $D M=$ deltóide médio]. 


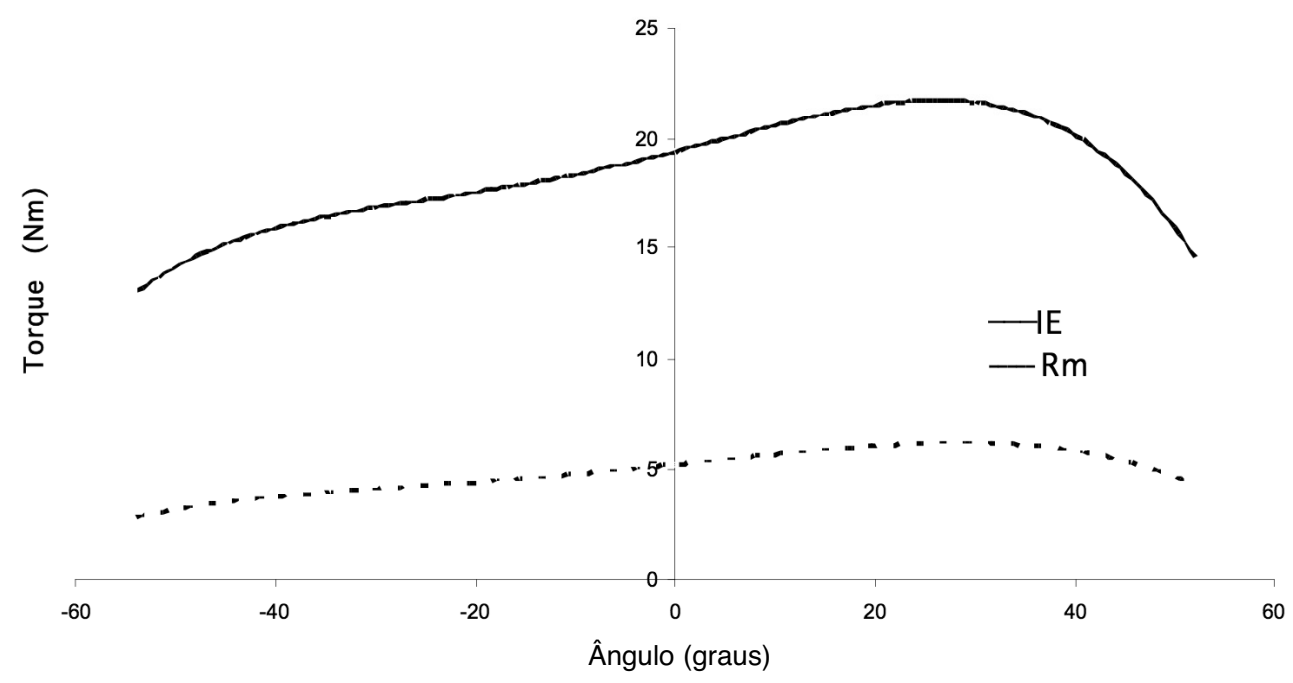

Figura 1. Torque Estimado dos Motores Primários de Rotação Externa.

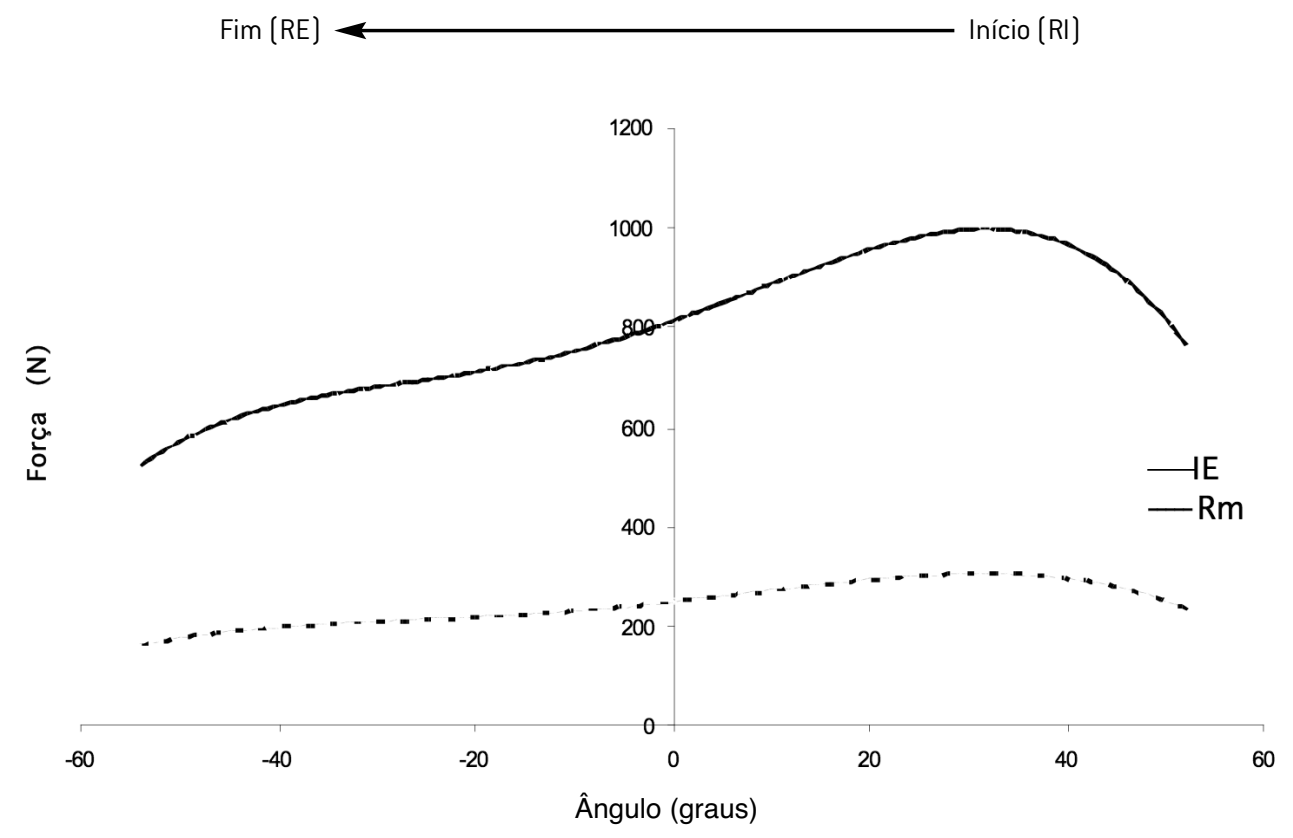

Figura 2. Força Estimada dos Motores Primários de Rotação Externa. 
A Tabela 1 apresenta a média, desvio padrão e os valores de pico com seus respectivos ângulos do torque e da força estimada de cada músculo, assim como os valores de torque potencial médio. $\mathrm{O}$ valor do fator limiar para determinação dos músculos motores primários e secundários foi de 5,7 Nm. Convencionou-se utilizar valores positivos de ângulo de rotação para expressar as posições de RI da articulação, enquanto valores negativos representam as posições de RE; a posição neutra da articulação é representada por zero grau no ângulo de rotação. O comportamento das curvas de torque dos músculos IE e Rm é similar (Figura 1), ambas possuem uma fase ascendente na amplitude inicial do movimento e rapidamente alcançam seus picos de $22 \mathrm{Nm} \mathrm{em} 20^{\circ} \mathrm{e}$ $6 \mathrm{Nm}$ em $28^{\circ}$. Após o pico, a curva possui um com- portamento descendente até o final do movimento. Na Figura 2, verifica-se que os músculos IE e Rm possuem curvas com comportamento ascendente no início do movimento, alcançando seus picos de 996 $\mathrm{N}$ e $306 \mathrm{~N}$, respectivamente, ambas em $31^{\circ}$. Estas curvas apresentaram um comportamento descendente até o final do gesto após alcançarem seus picos. A Figura 3a apresenta o comportamento das curvas de torque dos motores secundários de RE. Estes músculos possuem magnitudes de torque praticamente irrelevantes, não ultrapassando, em instante algum da ADM, 0,6 Nm. Já a Figura 3b apresenta o comportamento das curvas de força dos motores secundários. Existe um comportamento similar entre os três músculos (DP, SE e DM).

B Fim (RE)
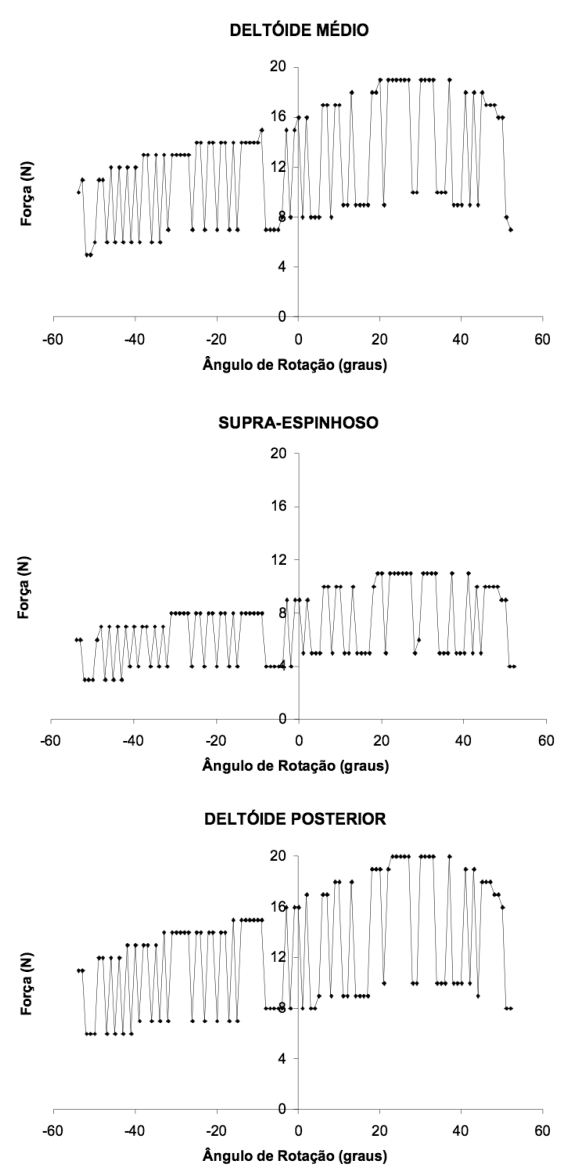

Figura 3. Torque Estimado dos Motores Secundários de Rotação Externa (A) e Força Estimada dos Motores Secundários de Rotação Externa (B]. 
A análise estatística verificou que os dados dos 6 conjuntos de valores que expressam as diferenças entre a produção de força e torque dos músculos IE e Rm, IE e SE e Rm e SE mostraram-se normais. A Tabela 2 apresenta as diferenças (diferença média, desvio padrão, pico/ângulo e valor estabelecido) encontradas entre: (a) IE e Rm, (b) IE e SE, e (c) $\mathrm{Rm}$ e SE para os dados de força e torque. O VE para IE e Rm mostra que o músculo IE apresenta uma produção de força maior que $500 \mathrm{~N}$, e uma produção de torque maior que $10 \mathrm{Nm}$, em relação ao $\mathrm{Rm}$, em cada grau da amplitude de movimento. Ou seja, se soubermos que o Rm produz $6 \mathrm{Nm}$ em uma determinada amplitude de movimento, o IE produziria, na mesma amplitude, no mínimo, $16 \mathrm{Nm}$. Quando o IE foi comparado ao SE, essas diferenças para os valores de força foram maiores que $700 \mathrm{~N}$ e de torque, maiores que $15 \mathrm{Nm}$. Já o músculo Rm apresentou diferenças de força maiores que $180 \mathrm{~N}$ e de torque, maiores que $4 \mathrm{Nm}$ quando comparado ao SE.

Tabela 2. Diferença de Força e Torque (Diferença Média, Desvio Padrão, Pico/Ângulo, Valor Estabelecido] entre os Músculos

\begin{tabular}{lccc}
\hline & IE - Rm & IE - SE & Rm - SE \\
\hline Torque (Nm) & & & \\
\hline Diferença Média & 13,57 & 18,9 & 4,84 \\
\hline Desvio Padrão & 1,48 & 2,35 & 0,91 \\
\hline Pico & 15,66 & 21,75 & 6,11 \\
\hline (Ângulo) & $\left(24^{\circ}\right)$ & $\left(25^{\circ}\right)$ & $\left(26^{\circ}\right)$ \\
\hline Valor Estabelecido & $10^{*}$ & $15^{*}$ & $4^{*}$ \\
\hline Força (N) & & & \\
\hline Diferença Média & 556,21 & 765,43 & 209,22 \\
\hline Desvio Padrão & 94,45 & 128,58 & 37,17 \\
\hline Pico & 690,78 & 963,41 & 273,39 \\
\hline (Ângulo) & $\left(31^{\circ}\right)$ & $\left(31^{\circ}\right)$ & $\left(29^{\circ}\right)$ \\
\hline Valor Estabelecido & $500^{*}$ & $700^{*}$ & $180^{*}$ \\
\hline
\end{tabular}

$(I \mathcal{E}=$ infra-espinhoso; $R m=$ redondo menor; $S \mathcal{E}=$ supra-espinhoso $) .{ }^{*} p<0,05$.

\section{DISCUSSÃO}

Segundo Kashima et al.(29) o movimento dinâmico de um membro pode ser determinado pelo torque que este produz, assim é possível analisar movimentos humanos avaliando o torque muscular. Os resultados de torque do grupo muscular, obtidos por meio do dinamómetro isocinético, mostram que os rotadores externos do ombro não produzem torque muscular de maneira constante ao longo da ADM e que as maiores magnitudes de torque encontram-se quando o ombro está posicionado em amplitudes de RI, quando os músculos encontram-se levemente alongados. Da mesma forma, no trabalho de Hughes et al.(25), houve maior capacidade de produzir torque por meio de contração isométrica com o ombro em RI de $30^{\circ}$ quando comparado à posição neutra. Estes dados corroboram com outros estudos que avaliaram a relação comprimento $\mathrm{x}$ tensão de estruturas mus-

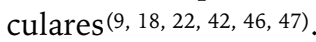

Os resultados deste estudo apontam que os músculos com maior capacidade de produzir torque de RE no ombro são o IE e o Rm, sucessivamente, sendo considerados motores primários. Estes resultados corroboram com os de Kuechle et al.(33), os quais relataram que estes músculos possuem as maiores magnitudes de $\mathrm{d}^{\wedge}$ durante toda ADM, reflectindo assim, em maior capacidade de produzir torque muscular.

Segundo Otis et al.(45), a $\mathrm{d}^{\perp}$ do músculo activo reflete se ele é funcionalmente motor primário ou estabilizador. Quando a linha de acção da força coincide com o centro de rotação da articulação ou passa muito próximo dele o músculo ao contrair-se gera apenas compressão e, consequentemente, tem a função de estabilizar a articulação. Nos resultados do presente estudo os músculos com menores $\mathrm{d}^{\perp}$ foram aqueles que possuíram menor capacidade de produzir torque (DP, SE e DM), sendo estes considerados motores secundários ou estabilizadores da articulação.

Visto que torque é o produto entre força muscular e $\mathrm{d}^{\perp}(14,24,32,37,45,48)$, as alterações das magnitudes de torque entre os músculos ocorreram principalmente devido às diferentes $\mathrm{d}^{\perp} \mathrm{e}$ capacidades de produção de força encontradas entre os músculos. Entretanto, analisando os resultados gerados pelo modelo de otimização, acredita-se que a variação na produção de torque ao longo da ADM (Figura 1) ocorra principalmente por influência da capacidade de produção de força (Figura 2), visto que, existem alterações discretas nas magnitudes de $d^{\perp}$ destes músculos durante o gesto estudado.

Para que ocorra contracção muscular, é necessária a excitação da unidade motora ${ }^{(29)}$. Desta forma, o número de unidades motoras activas e a frequência com que elas são estimuladas, são factores que influenciam a capacidade de produção de força mus- 
cular $^{(14,19)}$. Na medida em que foi solicitado aos indivíduos para realizarem esforço máximo ao longo de toda ADM, acredita-se que os indivíduos utilizaram toda sua capacidade durante a execução do gesto, sendo assim supõe-se que possivelmente tenha-se diminuído a influência do padrão de recrutamento na produção de força.

Outro factor que pode influenciar a capacidade de produção de força é a velocidade do gesto, ou seja, a relação força x velocidade. À medida que a velocidade do gesto aumenta, a capacidade de produção de força diminui $(4,14,16,39)$. Porém, acredita-se que este factor não tenha exercido interferência nas alterações da capacidade de produção de força dos músculos ao longo da ADM; isto porque o exercício foi realizado em um aparelho isocinético com velocidade constante $(60 \%$ s).

Os músculos considerados motores primários (IE e $\mathrm{Rm}$ ) apresentaram um comportamento descendente da curva de força à medida que se encurtavam, alcançando as menores magnitudes de força quando se encontravam mais encurtados (Figura 2).

Segundo Ruiter e Haan (10) a diminuição da capacidade de produção de força aumenta linearmente com a magnitude do encurtamento durante activação voluntária máxima, quando imposta uma velocidade constante. Assim como eles, diversos estudos têm relatado que a capacidade de produção de força muscular é diminuída com o encurtamento muscular(1, 7, 9, 11, 18, 20, 21, 41, 42, 46, 48).

Existem três hipóteses para justificar a diminuição da capacidade de produção de força após o encurtamento do músculo: a não uniformidade dos sarcômeros, o aumento da concentração de prótons e fosfato inorgânico no músculo e a inibição das pontes cruzadas por estresse induzido( ${ }^{(20)}$. Segundo Herzog e Leonard(20), por uma variedade de razões, a hipótese da inibição das pontes cruzadas é a mais aceita; desta forma podemos inferir que quando os músculos estudados encontravam-se mais encurtados (amplitudes de RE) ocorria uma sobreposição entre os filamentos de actina e miosina, proporcionando uma inibição das pontes cruzadas. Isto justificaria a diminuição da capacidade de produção de força nestas amplitudes. Por outro lado, o IE e o Rm apresentaram seus valores máximos de produção de força durante amplitudes de RI, quando se encontram alongados (Figura
2). Herzog et al.(22) e Rassier et al.(47) relataram que o pico de força de um músculo encontra-se onde seu sarcômero está um pouco mais alongado quando comparado a sua posição de repouso.

Segundo Rassier et al.(48) dois fatores devem ser observados ao avaliar o potencial de um músculo produzir movimento: $\mathrm{a}^{\perp}$ e o comprimento do músculo. Se a $\mathrm{d}^{\perp}$ é constante durante a ADM articular, a relação torque-ângulo reflecte a relação comprimento-tensão muscular. Desta forma, acredita-se que as alterações das magnitudes de força dos músculos IE e $\mathrm{Rm}$ ao longo da ADM ocorreram principalmente devido à relação comprimento $\mathrm{x}$ tensão.

Langenderfer et al.(35) realizaram um estudo em cadáveres onde calcularam a capacidade máxima de produção de torque isométrico de RE do ombro e concluíram que o IE é o músculo com maior capacidade para gerar torque. No algoritmo proposto por Favre et al.(15), o principal músculo a se opor a um torque de RI com o braço em $30^{\circ}$ de elevação e rotação neutra foi o IE, seguido pelo Rm. Da mesma forma, através de um modelo tridimensional, Hughes e An(24) encontraram os músculos IE e Rm como responsáveis pelo gesto de RE na posição neutra, apresentando as maiores magnitudes de força neste gesto. Em outro estudo, Langenderfer et al.(34) desenvolveram um modelo probabilístico onde investigaram a produção de força isométrica máxima dos RE com o ombro em posição neutra e em abdução de $30^{\circ}$. Em seus resultados, o músculo IE apresentou as maiores magnitudes de força seguida pelo SE e Rm. Ao contrário, os resultados do presente estudo sugerem uma pequena contribuição do SE para a produção do movimento, que provavelmente tenha acontecido devido suas baixas magnitudes de $\mathrm{d}^{\perp}$ ao longo da ADM.

Torna-se difícil comparar os resultados gerados pelo modelo de optimização do presente estudo com os resultados de outros trabalhos. Isto porque a maioria dos estudos encontrados na literatura utilizou análise estática $(6,15,24,28,36)$ e quando utilizaram análise dinâmica $(1,3,8,10,30,32,44)$, investigaram a actividade eletromiográfica. No entanto, dados eletromiográficos não podem determinar directamente produção de força muscular, já que o comprimento muscular, o tipo e a velocidade da contracção influenciam a relação entre EMG e força(38). 
Apesar da actividade eletromiográfica não representar a força que o músculo desenvolve, é possível utilizar estes dados para uma comparação qualitativa de resultados obtidos por modelos biomecânicos.

Alguns estudos que utilizaram EMG, relataram maior activação do músculo IE durante a RE do

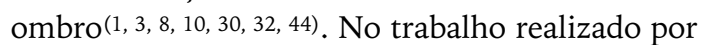
Hintermeister et al.(23) o músculo mais activo durante a RE com o braço em abdução de $45^{\circ} \mathrm{e}$ cotovelo em flexão de $90^{\circ}$, foi o IE, tendo sua maior activação quando se encontrava alongado. Da mesma forma, Kronberg et al.(32) avaliaram a activação muscular durante a RE com o ombro em posição neutral, em abdução de $45^{\circ}$ e $90^{\circ}$, encontrando em todas as posições o IE como músculo mais activo, além disso, o IE teve suas maiores magnitudes de activação quando se encontrava alongado, diminuindo à medida que se encurtava. Kelly et al.(30) estudaram a activação muscular em arremessadores e constataram que o músculo IE teve o maior sinal eletromiográfico nas primeiras fases do gesto, a qual, o principal movimento é a RE, já o DM e DP foram minimamente activos.

Inúmeros autores ressaltam o reforço dos músculos rotadores externos do ombro durante a reabilitação e preconizam um aumento progressivo da carga para estes músculos $(26,40,49,50)$. Entretanto, tais trabalhos não apresentam de forma clara em qual ADM devese começar o gesto(17, 26), e quando especificam, sugerem que os exercícios sejam iniciados na posição neutra $(10,31,40,49)$.

Se almejarmos submeter às estruturas musculares uma progressão gradual da carga, pode-se inferir baseando-se nos resultados gerados pelo modelo de optimização, que os exercícios para fortalecimento dos rotadores externos deverão iniciar com o ombro em ADM de RI (entre $40^{\circ}$ e $20^{\circ}$ ), progredindo gradualmente para amplitudes em que os músculos se encontrem mais encurtados, nas quais necessitam de maior esforço muscular para produção de uma mesma magnitude de força.

O modelo utilizado considera o gesto dinâmico como uma sequência de posições estáticas, desconsiderando as propriedades inerciais dos segmentos, entretanto, o movimento foi realizado em um dinamômetro isocinético onde acredita-se que os efeitos das acelerações e desacelerações são diminuídos, uma vez que a velocidade é controlada, restringindo assim, a influência das propriedades inerciais sobre os segmentos corporais. Além disso, o modelo possui outras limitações, tais como: a análise de força foi realizada em apenas um plano de movimento, não foi considerado a força de contacto articular da gleno-umeral; não levou-se em consideração movimentos escapulares, o modelo não é capaz de prever co-contracção nem força muscular para prover estabilização. Mais estudos utilizando modelos de optimização e analisando outros gestos são necessários para compreendermos melhor a contribuição que cada músculo fornece para a geração do torque muscular e para guiar a elaboração de exercícios em programas de reabilitação.

\section{CONCLUSÃO}

Os resultados gerados pelo modelo de optimização nos permitem analisar a contribuição de cada músculo para a produção de torque e força muscular de RE do ombro. Estes resultados permitem admitir que os músculos IE e Rm são preferencialmente utilizados para o gesto de RE do ombro, enquanto que os músculos DP, SE e DM contribuem com magnitudes discretas de força e torque muscular, provavelmente com o objetivo de estabilizar a articulação.

\section{CORRESPONDÊNCIA}

\section{Marcelo P. Castro}

Rua Botafogo 1212/501

CEP: 90150052

Porto Alegre - RS, Brasil.

E-mail: marcelocastro_fisio@hotmail.com 


\section{REFERÊNCIAS}

1. Abbott BC, Aubert XM (1952). The force exerted by active striated muscle during and after change of length. J Physiol 117(1): 77-86.

2. An K, Kaufmn K, Eys C (1995). Estimation of muscle and joint forces. In: Three-Dimensional Analysis of Human Movement, Champaign: Human Kinetics, 201-214.

3. Ballantyne BT, O'Hare SJ, Paschall J, Pavia-Smith MM, Pitz AM, Gillon JF., Soderberg GL (1993). Electromyographic activity of selected shoulder muscles in commonly used therapeutic exercises. Phys Ther 73(10): 668-677; discussion 677-682.

4. Bottinelli R, Canepari M, Pellegrino MA, Reggiani C (1996). Force-velocity properties of human skeletal muscle fibres: myosin heavy chain isoform and temperature dependence. J Physiol 495 ( Pt 2): 573-586.

5. Buchanan TS (1995). Evidence that maximum muscle stress is not a constant: differences in specific tension in elbow flexors and extensors. Med Eng Phys, 17(7): 529-536.

6. Chang YW, Hughes RE, Su FC, Itoi E, An KN (2000). Prediction of muscle force involved in shoulder internal rotation. J Shoulder Elbow Surg 9(3):188-95.

7. Corr DT, Herzog W (2005). Force recovery after activated shortening in whole skeletal muscle: transient and steadystate aspects of force depression. J Appl Physiol 99(1): 252 260.

8. David G, Magarey ME, Jones MA, Dvir Z, Turker KS, Sharpe M (2000). EMG and strength correlates of selected shoulder muscles during rotations of the glenohumeral joint. Clin Biomech (Bristol, Avon) 15(2): 95-102.

9. De Ruiter CJ, De Haan A (2000). Temperature effect on the force/velocity relationship of the fresh and fatigued human adductor pollicis muscle. Pflugers Arch 440(1): 163170.

10. De Ruiter CJ, De Haan A (2003). Shortening-induced depression of voluntary force in unfatigued and fatigued human adductor pollicis muscle. J Appl Physiol 94(1): 6974.

11. De Ruiter CJ, De Haan A, Jones DA, Sargeant AJ (1998). Shortening-induced force depression in human adductor pollicis muscle. J Physiol 507 ( Pt 2): 583-591.

12. De Wilde LF, Audenaert EA, Berghs BM (2004). Shoulder prostheses treating cuff tear arthropathy: a comparative biomechanical study. J Orthop Res 22(6): 1222-1230.

13. Edman KA (1975). Mechanical deactivation induced by active shortening in isolated muscle fibres of the frog. $J$ Physiol 246(1): 255-275.

14. Enoka RM (2000). Bases Neuro Mecânicas da Cinesiologia, $2^{\text {a }}$ Ed., Editora Manole Ltda, São Paulo.

15. Favre P, Sheikh R, Fucentese SF, Jacob HA (2005). An algorithm for estimation of shoulder muscle forces for clinical use. Clin Biomech (Bristol, Avon) 20(8): 822-833.

16. Fenn and Marsh. (1935). Muscular Force at Different Speeds of Shortening. Department of Physiology - The University of Rochester School of Medicine - NY.

17. Glasoe WM, Fisher CJ, Murthy D (2004). Treatment protocol for an acute large rotator cuff repair. Physiotherapy 90: 217-220.

18. Gordon AM, Huxley AF, Julian FJ (1966). The variation in isometric tension with sarcomere length in vertebrate muscle fibres. J Physiol 184(1): 170-192.

19. Guyton AC, Hall JE (2002). Tratado de fisiologia médica, $10^{\mathrm{a}}$
Ed., Guanabara Koogan, Rio de Janeiro.

20. Herzog W, Leonard TR (1997). Depression of cat soleusforces following isokinetic shortening. J Biomech 30(9): 865-872.

21. Herzog W, Leonard TR, Wu JZ (2000). "The relationship between force depression following shortening and mechanical work in skeletal muscle." J Biomech 33(6): 659-668.

22. Herzog W, Schachar R, Leonard TR (2003). Characterization of the passive component of force enhancement following active stretching of skeletal muscle. J Exp Biol 206 (Pt 20): 3635-3643.

23. Hintermeister RA, Lange GW, Schultheis JM, Bey MJ, Hawkins RJ (1998). Electromyographic activity and applied load during shoulder rehabilitation exercises using elastic resistance. Am J Sports Med 26(2): 210-220.

24. Hughes RE, An KN (1996). Force analysis of rotator cuff muscles. Clin Orthop Relat Res (330): 75-83.

25. Hughes RE, Johnson ME, O’Driscoll SW, An KN (1999). Age-related changes in normal isometric shoulder strength. Am J Sports Med 27(5): 651-657.

26. Jobe FW, Moynes DR, Brewster CE (1987). Rehabilitation of shoulder joint instabilities. Orthop Clin North Am 18(3): 473-482.

27. Kapandje IA (2000). Fisiologia Articular $5^{\text {a }}$ Ed., Panamericana, São Paulo.

28. Karlsson D, Peterson B (1992). Towards a model for force predictions in the human shoulder. J Biomech 25(2): 189199.

29. Kashima T, Isurugi Y, Shima M (2000). Analysis of a muscular control system in human movements. Biol Cybern 82(2): 123-131.

30. Kelly BT, Backus SI, Warren RF, Williams RJ (2002). Electromyographic analysis and phase definition of the overhead football throw. Am J Sports Med 30(6): 837-844.

31. Kibler WB, McMullen J, Uhl T (2001). Shoulder rehabilitation strategies, guidelines, and practice. Orthop Clin North Am 32(3): 527-538.

32. Kronberg M, Nemeth G, Brostrom LA (1990). Muscle activity and coordination in the normal shoulder. An electromyographic study. Clin Orthop Relat Res (257): 76-85.

33. Kuechle DK, Newman SR, Itoi E, Niebur GL, Morrey BF, An KN (2000). The relevance of the moment arm of shoulder muscles with respect to axial rotation of the glenohumeral joint in four positions. Clin Biomech (Bristol, Avon) 15(5): 322-329.

34. Langenderfer JE, Carpenter JE, Johnson ME, An KN, Hughes RE (2006). A probabilistic model of glenohumeral external rotation strength for healthy normals and rotator cuff tear cases. Ann Biomed Eng 34(3): 465-476.

35. Langenderfer JE, Patthanacharoenphon C, Carpenter JE, Hughes RE (2006). Variability in isometric force and moment generating capacity of glenohumeral external rotator muscles. Clin Biomech (Bristol, Avon) 21(7): 701709.

36. Laursen B, Jensen BR, Nemeth G, Sjogaard G (1998). A model predicting individual shoulder muscle forces based on relationship between electromyographic and 3D external forces in static position. J Biomech 31(8): 731-739.

37. Liu J, Hughes RE, Smutz WP, Niebur G, Nan-An K (1997). Roles of deltoid and rotator cuff muscles in shoulder elevation. Clin Biomech (Bristol, Avon) 12(1): 32-38.

38. Ludewig PM, Cook TM (2000). Alterations in shoulder 
kinematics and associated muscle activity in people with symptoms of shoulder impingement. Phys Ther 80(3): 276291.

39. MacIntosh BR, Herzog W, Suter E, Wiley JP, Sokolosky J (1993). Human skeletal muscle fibre types and force: velocity properties. Eur J Appl Physiol Occup Physiol 67(6): 499506.

40. Mantone JK, Burkhead WZ Jr., Noonan J Jr. (2000). Nonoperative treatment of rotator cuff tears. Orthop Clin North Am 31(2): 295-311.

41. Marechal G, Plaghki L (1979). The deficit of the isometric tetanic tension redeveloped after a release of frog muscle at a constant velocity. J Gen Physiol 73(4): 453-467.

42. Morgan, D. L., Whitehead, N. P., Wise, A. K., Gregory, J. E., and Proske, U. (2000). "Tension changes in the cat soleus muscle following slow stretch or shortening of the contracting muscle." J Physiol, 522 Pt 3, 503-513.

43. Murray WM, Delp SL, Buchanan TS (1995). Variation of muscle moment arms with elbow and forearm position. $J$ Biomech 28(5): 513-525.

44. Nieminen H, Niemi J, Takala EP, Viikari-Juntura E (1995). Load-sharing patterns in the shoulder during isometric flexion tasks. J Biomech 28(5): 555-566.

45. Otis JC, Jiang CC, Wickiewicz TL, Peterson MG, Warren RF, Santner TJ (1994). Changes in the moment arms of the rotator cuff and deltoid muscles with abduction and rotation. J Bone Joint Surg Am 76(5): 667-676.

46. Rassier DE, Herzog W (2004). Considerations on the history dependence of muscle contraction. J Appl Physiol 96(2): 419-427.

47. Rassier DE, Herzog W, Pollack GH (2003). Dynamics of individual sarcomeres during and after stretch in activated single myofibrils. Proc Biol Sci 270(1525): 1735-1740.

48. Rassier DE, MacIntosh BR, Herzog W (1999). Length dependence of active force production in skeletal muscle. $J$ Appl Physiol 86(5): 1445-1457.

49. Rubin BD, Kibler WB (2002). Fundamental principles of shoulder rehabilitation: conservative to postoperative management. Arthroscopy 18(9 Suppl 2): 29-39.

50. Tytherleigh-Strong G, Hirahara A, Miniaci A (2001). Rotator cuff disease. Curr Opin Rheumatol 13(2): 135-145.

51. Vecchia ED, Duarte M, Amadio AC (1997). Proposta de um modelo metodológico para determinação de forças internas do aparelho locomotor humano. Anais do VII Congresso Brasileiro de Biomecânica, Campinas, 189-194.

52. Wilmore J, Costill D (2001). Fisiologia do Esporte e do Exercício, Manole, São Paulo.

53. Wood JE, Meek SG, Jacobsen SC (1989). Quantitation of human shoulder anatomy for prosthetic arm control-I. Surface modelling. J Biomech 22(3): 273-292. 